\title{
Nominata de Avaliadores
}

Agradecemos a todos os avaliadores ad hoc, listados a seguir, que estiveram à disposição da Revista Research, Society and Development durante o ano de 2016:

\begin{tabular}{|c|c|c|}
\hline Nome & Instituição & País \\
\hline Acsa Nara de Araújo Brito & Universidade Federal do Rio Grande do Norte & Brasil \\
\hline Adriam Camacho Domínguez & Universidad Laica Vicente Rocafuerte de Guayaquil & Equador \\
\hline Alex Paubel Junger & Universidade Nove de Julho & Brasil \\
\hline Bruno Carpinetti & Universidad Nacional Arturo Jauretche & Argentina \\
\hline Carlos Anibal Peris Castiglioni & Universidad Nacional de Asunción & Paraguai \\
\hline Carlos Eymel Campos Rodriguez & Universidade Federal de Itajubá & Brasil \\
\hline Carmino Hayashi & Universidade Federal do Triângulo Mineiro & Brasil \\
\hline Clegiane Santos Bezerra Dantas & Secretaria de Educação e Cultura do RN & Brasil \\
\hline Desiré Nguessan & Universidade de Mogi das Cruzes & Brasil \\
\hline Eugenio Gustavo Coconier & Universidad de Buenos Aires & Argentina \\
\hline Felix Raúl España Cuellar & Universidad Mayor de San Andres & Bolívia \\
\hline Freddy Oropeza & Universidad Pedagógica Experimental Libertador & Venezuela \\
\hline Gessica Fabiely Fonseca & Universidade Federal do Rio Grande do Norte & Brasil \\
\hline Gleicemares Maria Marques & Universidade Federal de Itajubá & Brasil \\
\hline Henry Anibal Angulo Serrano & Universidad Católica Boliviana San Pablo & Bolívia \\
\hline Jael Glauce Fonseca & Universidade Federal da Bahia & Brasil \\
\hline Josefa da Conceição Silva & Universidade Federal de Roraima & Brasil \\
\hline José Blas Villalba Giménez & Universidad del Cono Sur de las Américas & Paraguai \\
\hline José Rubens Damas Garlipp & Universidade Federal de Uberlândia & Brasil \\
\hline Konstantinos Ravanis & University of Patras & Grécia \\
\hline Loan José Landaeta & Universidad Pedagógica Experimental Libertador & Venezuela \\
\hline Luiz Arnaldo Fernandes & Universidade Federal de Minas Gerais & Brasil \\
\hline Luis Camilo Kunstek Salinas & Universidad Católica Boliviana San Pablo & Bolívia \\
\hline Maximiliano Bezada Díaz & Universidad Pedagógica Experimental Libertador & Venezuela \\
\hline Max Leandro de Araújo Brito & Universidade Federal de Itajubá & Brasil \\
\hline
\end{tabular}


Research, Society and Development, v. 3, n. 2, p. 188-189, set. 2016

\begin{tabular}{|l|l|c|}
\hline \multicolumn{1}{|c|}{ Nome } & \multicolumn{1}{c|}{ Instituição } & País \\
\hline Mayara Mirela Balbino de Souza & Universidade Federal do ABC & Brasil \\
\hline Orlando José González Clemente & Universidad Pedagógica Experimental Libertador & Venezuela \\
\hline Orivaldo de Lira Tavares & Universidade Federal do Espírito Santo & Brasil \\
\hline Paulo César Pontes Fraga & Universidade Federal de Juiz de Fora & Brasil \\
\hline Priscilla Chantal Duarte Silva & Universidade Federal de Itajubá & Brasil \\
\hline Raquel Fernandes de Macedo & Universidade Federal do ABC & Brasil \\
\hline Rhodiney Vaz Martins & Universidade Virtual do Estado de São Paulo & República \\
\hline Rodrigo Leonardo Guillén Bretón & Universidad del Caribe & Dominicana \\
\hline Ronara Cristina Bozi dos Reis & Universidade Federal de Itajubá & Brasil \\
\hline Ruan Kléberson Pereira da Silva & Universidade Federal do Rio Grande do Norte & Brasil \\
\hline Sergio Foghin Pillin & Universidad Pedagógica Experimental Libertador & Venezuela \\
\hline Víctor Hugo Mamani Yapura & Universidad Indígena Quechua Casimiro Huanca & Bolívia \\
\hline Weimar Giovanni Iño Daza & Universidad de Jaume I & Espanha \\
\hline
\end{tabular}

Dr. Ricardo Shitsuka

Editor 